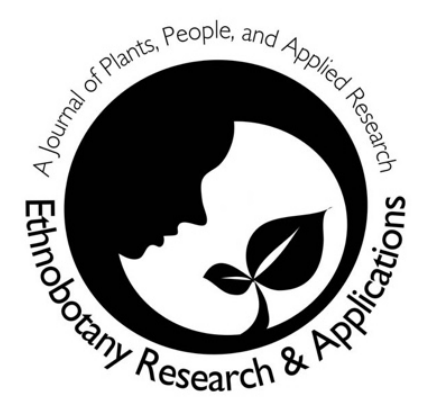

\title{
Current knowledge encounters in ethnobiological studies fit equilibrium systems
}

\author{
Mónica Moraes R.
}

\section{Editorial}

"Indigenous peoples and local communities possess detailed knowledge on biodiversity and ecosystem trends. This knowledge is formed through their direct dependence on their local ecosystems, and observations and interpretations of change generated and passed down over many generations, and yet adapted and enriched over time. Indigenous peoples and local communities from around the world often live in remote areas, interacting with nature and managing resources that contribute to society at large. They also suffer directly from the pressures of expanding agriculture frontiers and commodity production, such as mining, logging, and energy. They are often better placed than scientists to provide detailed information on local biodiversity and environmental change and are important contributors to the governance of biodiversity from local to global levels." Intergovernmental Panel on Biodiversity and Ecosystem Services" (IPBES)

The field work performance of ethnobiological studies with human communities has covered very contrasting trajectories and a chronology that reaches the present, which is nourished by more experiences in research networks and accordingly to a closer collaboration level. In general, efforts are being made to reverse old 'exhaustive knowledgeextractive' practices that favor mostly one of the parties, a long list of discontinued studies, not fulfilled offers by people and institutions, among others. In this contribution I will summarize some perceptions derived from recent ethnobiological studies carried out in Bolivia, in response to regional challenges and consensus of scientists who have promoted an increasingly fortified content.

Several organizations have discussed several scenarios in which science, nature and human communities converge. International organizations, for example, thematic regulations in the United Nations Convention on Biological Diversity, the Determination of Peoples, the Nagoya Protocol and the operation of cooperation networks, such as the Latin American Society of Ethnobiology (SOLAE) have convened to several actors. In general, efforts are made to adjust and include recommendations to apply better procedures. All the guidelines, concepts and recommendations have had meeting points that, of course, favor collaborative studies. An example at the regional level was the project "Access and benefit-sharing in Latin America and the Caribbean: a dialogue between science and policy in the field of academic research" carried out during 2013 by Diversitas, the Regional Office for Latin America and the Caribbean. Caribbean of the International Organization. Council for Science (ICSU-ROLAC), the Swiss Academy of Sciences (SCNAT) and the International Union of Biological Sciences (IUBS). The objective was to collect information on the key elements of access and benefit sharing for scientific research in the Latin America and the Caribbean region.

\begin{tabular}{l} 
Correspondence \\
Mónica Moraes R. \\
Herbario Nacional de Bolivia, Instituto de \\
Ecología, Facultad de Ciencias Puras y \\
Naturales, Universidad Mayor de San Andrés, \\
Calle 27 Cota Cota s / n campus universitario \\
La Paz, Bolivia \\
mmoraes@fcpn.edu.bo \\
Ethnobotany Research \& Applications \\
$\mathbf{1 7 : 2 ~ ( 2 0 1 8 )}$ \\
\hline
\end{tabular}

During the organized workshops, both components, the academic and the politician in governmental instances took place and discussed on different topics; Case studies from eight countries were 
included in the compiled document. As part of the final report, they were substantially summarized in the institutional implementation of the access and benefit distribution system, as well as favorable conditions and opportunities prior to the research process.

In addition, the United Nations Intergovernmental Panel on Biodiversity and Ecosystem Services (IPBES), which was created in April 2012, is mandated to assess the state of the planet's biodiversity and ecosystems and provide scientific information accessible to policy makers. Indigenous and local knowledge (ILK) is intended to support the achievement of product 1 (c) of the work program, in relation to procedures and approaches to work with local and indigenous knowledge systems. Among its responsibilities are the following (https://www.ipbes.net/indigenous-local-knowledgemandate): Oversee the development of procedures and approaches to work with local and indigenous knowledge systems, including the convening of workshops global dialogue and development. case studies; undertake work to facilitate the contribution of indigenous and local knowledge systems, in particular to test preliminary procedures and approaches for working with indigenous and local knowledge systems in accelerated, thematic, regional and sub-regional assessments; advise on the establishment of a list and a network of indigenous and local knowledge experts to support the work of the Panel; support the establishment of a participatory mechanism for indigenous and local knowledge systems to facilitate linkages between indigenous and local communities and scientists; and liaise with the task force on capacity-building and the knowledge and data team to ensure that they address issues related to local and cultural knowledge as a counterpart and complementary approaches.

The organization of events such as the ethnobiology colloquium also plays a fundamental role in the meeting of various areas of Bolivian knowledge and researchers: biologists, botanists, agronomists, archaeologists, sociologists, anthropologists and others. At present in Bolivia we have had two colloquia since 2016 and the exchange has delivered interesting research topics that are in progress. The recommendations and conclusions were presented in manifestos to increase the level of disclosure to a wider audience. It is expected to continue with this line and seek to diversify the participation of local actors and target groups of our research with human communities.
Although some unfavorable examples are still known for the trajectory of ethnobiological projects, many efforts and the best dedication of recognition to the knowledgeable and wise members of human communities in terms of their cultures and traditions are concentrated in several drafts and proposals of ethical codes. These codes became tools that guide and substantially try to generate an environment of equity and shared opportunities for joint work involving scientists and human communities; a clear example is the Code of ethics for ethnobiological research in Latin America (http://www.ethnoscientia.com/index.php/revista/arti cle/view/174) published in 2018. By establishing spaces for the previous presentation, free and informed of the project that will be developed with its scope, activities and products, the conditions were also opened and strengthened to establish collaboration and counterpart agreements, between the wise people of the indigenous people and the scientists. Then, counterpart pacts were generated that illustrated the validity of the project based on the contributions of both parties. Depending on each project, the assigned tasks and competencies are distributed according to the capacities and learning of those who form these cooperative relationships. Commitments and responsibilities are also included to contribute to the activities and products of the project.

In practical terms, each experience seeks to optimize the interaction established in the research carried out with the support and collaboration of human communities and peoples. As preliminary results and with a wide impact is that the systems of analysis between scientists and communities are adapted; the exchange of knowledge generates a richer and more complete option. Among the most convincing results that derive from these collaborative studies, is the point of analysis of both parties: based on cultural knowledge and how they use natural environments versus scientific knowledge based on protocols and standardized schemes that systematize data and consolidate a horizontal scenario The points of view are also harmonized in a complementary fusion of both capacities. Even more important is that new and more solid lines of cooperation are established and research projects are governed by a continuous sequence of evaluations, which enrich the whole analysis, the rescue of traditions and the options of labor complementarity through participatory workshops and information exchange

The orientation derived from these ethnobiological investigations in Bolivia is added to the cultural and traditional environment to also involve the producers 
and gatherers of the products of nature, who have not necessarily inhabited these spaces in capacity or historical knowledge, but rather recently. They are new immigrants in the country for less than 10 years and do not know those natural landscapes adequately. Therefore, in the processes of delivering their knowledge, the results of certain studies result in a guide and different types of manuals to correct errors, change hostile practices with sustained production and highlight positive procedures. We have advanced more and developed scenarios with a broad exchange of knowledge, but surely we still need to establish collaborative work windows where knowledge is horizontal and complemented. Each time the stages consolidate those bonds under consensus, respect and joint participation. 